\title{
Breastfeeding-associated microbiota in human milk following supplementation with Lactobacillus rhamnosus GG, Lactobacillus acidophilus La-5, and Bifidobacterium animalis ssp. lactis Bb-12
}

\author{
Melanie Rae Simpson, ${ }^{* 1}$ Ekaterina Avershina,† Ola Storrø, ${ }^{*}$ Roar Johnsen, ${ }^{*}$ Knut Rudi, $\dagger$ and Torbjørn Øien* \\ *Department of Public Health and Nursing, NTNU - Norwegian University of Science and Technology, N-7030 Trondheim, Norway \\ †Department of Chemistry, Biotechnology and Food Science, University of Life Sciences, N-1432 Ås, Norway
}

\begin{abstract}
Breastfeeding is one of the major factors affecting the early development of the infant gut microbiota, and weaning is associated with a shift in the gut microbiota toward a more adult composition. Through breastfeeding, infants receive bioactive components that shape their microbiota while also being exposed to the breast milk and breast surface microbial communities. Recent studies have suggested the possibility of an enteromammary route of microbial transfer, opening the possibility of infant gut microbiota modulation through maternal probiotic supplementation. In this study, we have analyzed breast milk samples collected at $10 \mathrm{~d}$ and 3 mo postpartum from women participating in the Probiotics in the Prevention of Allergy among Children in Trondheim placebo controlled trial. Women who were randomized to the probiotic arm of the Probiotics in the Prevention of Allergy among Children in Trondheim trial received a fermented milk supplemented with Lactobacillus rhamnosus GG, Lactobacillus acidophilus La-5, and Bifidobacterium animalis ssp. lactis Bb-12, consuming this daily from $4 \mathrm{wk}$ before their expected due date until 3 mo after birth. In total, 472 breast milk samples were assessed for the administered bacteria using quantitative real-time PCR and the microbiota transferred during breastfeeding was analyzed using $16 \mathrm{~S}$ ribosomal RNA gene sequencing of 142 samples. We found that breastfeeding is unlikely to be a significant source of L. rhamnosus GG, L. acidophilus La-5, and B. animalis ssp. lactis $\mathrm{Bb}-12$ for infants in the probiotic arm of the trial. Furthermore, maternal supplementation did not significantly affect the overall composition of the breast milk microbiota transferred during breastfeeding. We also present a descriptive analysis of
\end{abstract}

Received June 29, 2017.

Accepted October 17, 2017.

${ }^{1}$ Corresponding author: melanie.simpson@ntnu.no this microbiota, which was largely dominated by Streptococcus and Staphylococcus genera at both $10 \mathrm{~d}$ and 3 mo postpartum. Samples collected at 3 mo postpartum had a statistically significant lower presence and relative abundance of the Staphylococcus genus. These samples also had a greater number of observed species and diversity, including more operational taxonomic units from the Rothia, Veillonella, Granulicatella, and Methylbacterium genera.

Key words: human milk, probiotics, atopic dermatitis, microbiota

\section{INTRODUCTION}

Breastfeeding is one of the major factors affecting the early development of the infant gut microbiota and weaning is associated with a shift in the gut microbiota toward a more adult-like composition (Wopereis et al., 2014; Bäckhed et al., 2015; Rodríguez et al., 2015). Multiple components of breast milk contribute to these effects, including human milk oligosaccharides, which promote the growth of some microbes, and lysozymes, lactoferrin, and antimicrobial peptides, which inhibit the growth of others (Cacho and Lawrence, 2017). Additionally, breastfeeding is a source of a diverse range of microbes that are found both on the breast surface and within the mammary glands of lactating women (Fitzstevens et al., 2017). Culture-dependent and -independent techniques have demonstrated a dominance of bacteria belonging to the Staphylococcus, Streptococcus, and Propionibacterium genera, as well as the presence of lactic acid bacteria and bifidobacteria in breast milk (Fitzstevens et al., 2017). The origin of these bacteria is thought to be a combination of the microbiotas associated with the mother's skin flora, the infant's oral mucosa, and the maternal gut. Recent studies have suggested the possibility of an entero-mammary route with selective trafficking of commensal bacteria from the maternal gut to the mammary glands via dendritic cells and macrophages (Rodríguez, 2014; Treven et al., 2015). 
In the Probiotics in the Prevention of Allergy among Children in Trondheim (ProPACT) placebo controlled trial, we found that maternal ingestion of 3 probiotic strains, while pregnant and breastfeeding, resulted in an almost $40 \%$ reduction in the cumulative incidence of atopic dermatitis among offspring at $2 \mathrm{yr}$ of age (Dotterud et al., 2010). Women in the probiotic arm of the trial consumed fermented milk supplemented with Lactobacillus rhamnosus GG (LGG), Lactobacillus acidophilus La-5 (La-5), and Bifidobacterium animalis ssp. lactis Bb-12 (Bb-12), whereas women in the placebo group consumed heat-treated fermented milk without supplemented bacteria. Analysis of stool samples from the ProPACT study revealed that women in the probiotic arm had a higher prevalence and relative abundance of all 3 probiotic bacteria strains at 3 mo postpartum in their stool samples. A similar result was observed for the children of these women at $10 \mathrm{~d}$ and 3 mo of age, although only for the LGG (Dotterud et al., 2015). Breastfeeding may therefore be an ongoing source of LGG for these infants. Previous studies suggest that maternal supplementation with L. rhamnosus LC705 (Nasiraii et al., 2011), Lactobacillus reuteri (Abrahamsson et al., 2009), and Lactobacillus fermentum CECT5716 and Lactobacillus salivarius CECT5713 (Arroyo et al., 2010) may result in an increased presence of the administered bacteria in the breast milk of some, but not all, women. To our knowledge, no studies have investigated the transfer of LGG, L. acidophilus, or Bifidobacterium species through breastfeeding after maternal supplementation. Lactobacillus rhamnosus GG is of particular interest because it has been the most commonly administered bacteria in atopic dermatitis prevention studies and was observed to be transferred to infants in the ProPACT study.

In the present study, we investigated the bacteria transferred through breastfeeding using breast milk samples taken at $10 \mathrm{~d}$ and 3 mo postpartum from women participating in the ProPACT trial. The samples were collected without sterilization of the breast areola and are considered to give a more representative analysis of the bacteria ingested by suckling infants. We have therefore adopted the term "breastfeeding-associated microbiota" suggested by Sakwinska et al. (2016) to describe this bacterial community, which involves the breast milk and breast surface microbiotas in human milk ingested by the suckling infant. Our aim was to investigate whether maternal probiotic supplementation with LGG, La-5, and Bb-12 affected the presence of these strains among the bacteria transferred during breastfeeding at $10 \mathrm{~d}$ and 3 mo postpartum, and their association with the later development of atopic dermatitis. We also assessed the general microbiota associ- ated with breastfeeding, considered temporal trends, and the relationships between the composition of the microbiota transferred during breastfeeding, probiotic supplementation, and atopic dermatitis.

\section{MATERIALS AND METHODS}

\section{Participant Recruitment and Sample Collection}

This study analyzed 472 breast milk samples collected from 252 women participating in the ProPACT trial. The design and clinical results from this randomized, placebo controlled trial have been described in detail elsewhere (Dotterud et al., 2010; Simpson et al., 2015). Briefly, 415 women, who intended to breastfeed, were randomized to receive a commercially available fermented milk (Biola, Tine AS, Oslo, Norway) containing $5 \times 10^{10} \mathrm{cfu}$ of LGG and Bb-12 and $5 \times 10^{9} \mathrm{cfu}$ of La-5 per $250 \mathrm{~mL}$ or a placebo fermented milk that contained no probiotic bacteria and was heat treated after fermentation. Participating women were to consume $250 \mathrm{~mL}$ per day of their allocated study milk from 36 wk gestation until 3 mo postpartum. Their infants did not receive any probiotic supplementation. The children were assessed for signs and symptoms of allergy-related diseases through questionnaires and clinical examination at 2 and 6 yr of age. Atopic dermatitis was diagnosed according to the UK Working Party diagnostic criteria (Williams et al., 1994) at the clinical examinations.

Participating women were provided with sterile sample tubes and were requested to collect breast milk at $10 \mathrm{~d}$ and 3 mo postpartum. The timing, with respect to time of day or whether fore- or hindmilk was collected, was not standardized. The women did not receive explicit instructions regarding washing or sterilization of the breast surface before sample collection. Samples were frozen in their home freezer until transportation to the laboratory where they were subsequently stored at $-80^{\circ} \mathrm{C}$. All available breast milk samples were included in the current study, provided that the child attended the 2-yr clinical follow-up (Figure 1).

\section{Analysis of Microbiota}

Breast milk samples $(2 \mathrm{~mL})$ were centrifuged at $21,500 \times g$ for $30 \mathrm{~min}$. The resulting pellet was resuspended in $100 \mu \mathrm{L}$ of stool transport and recovery buffer and DNA was isolated using LGC Mag DNA extraction kit (LGC Genomics, Middlesex, UK) on a KingFisher FLEX magnetic particle processor (Thermo Fisher Scientific, Waltham, MA) according to the manufacturers' instructions. Samples were analyzed for total bacteria 


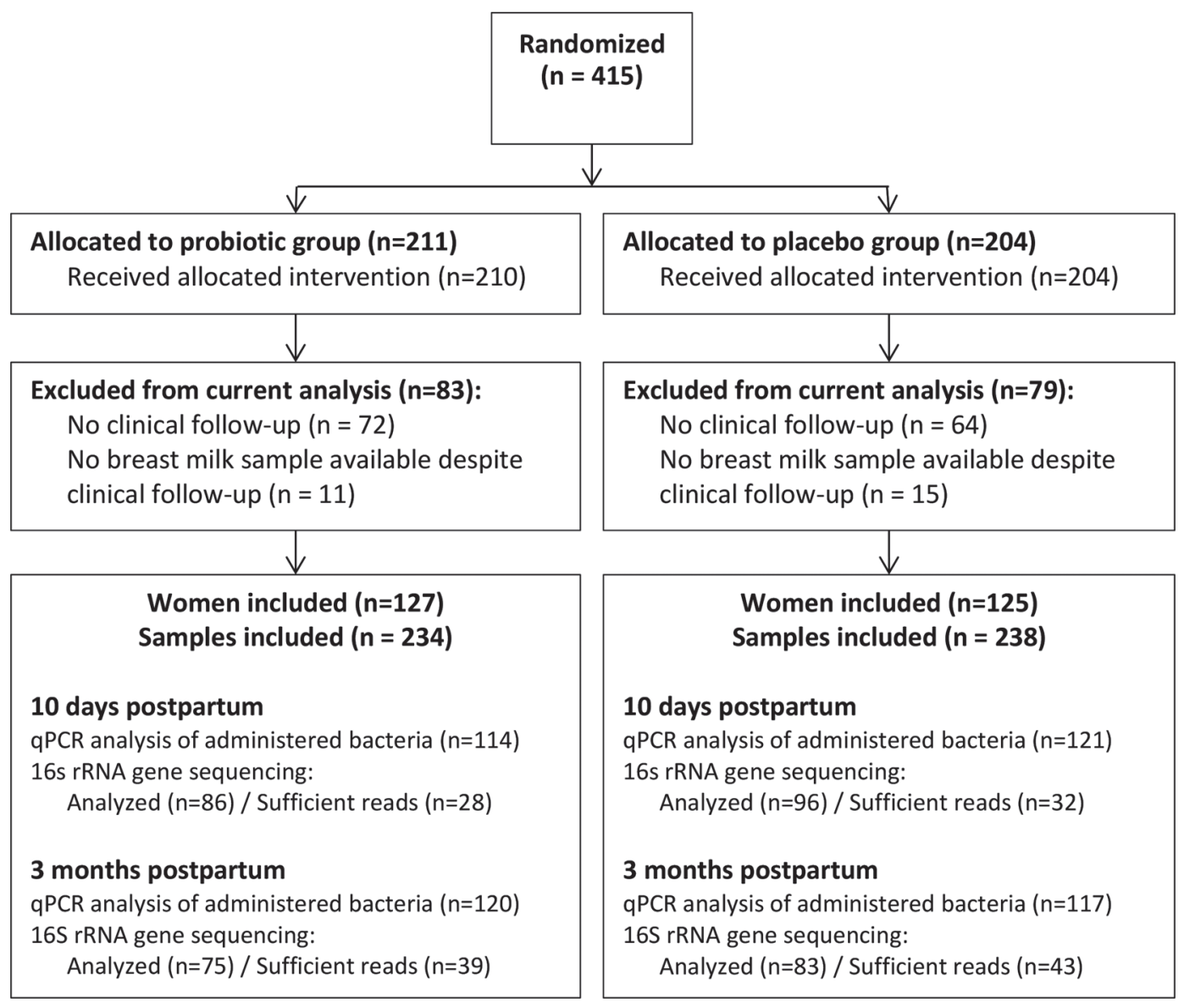

Figure 1. Flow diagram of participants in the Probiotics in the Prevention of Allergy among Children in Trondheim study and analysis of the bacterial content of breast milk samples is given. The number of eligible women who had provided at least one breast milk sample. The precise number of samples analyzed at each time point with each method varies as indicated. Samples were considered to have a sufficient number of reads if the $16 \mathrm{~S}$ rRNA gene sequencing analysis returned $\geq 1,000$ reads. $\mathrm{qPCR}=$ quantitative $\mathrm{PCR}$.

and the 3 administered bacteria using quantitative realtime PCR (qPCR) and using 16S rRNA gene sequencing for the general microbial composition.

The qPCR was performed using a double-stranded DNA binding dye-based EvaGreen assay (Biotium Inc., Fremont, CA) for assessing LGG and La5, and a probe-based TaqMan assay (Thermo Fisher Scientific) for Bb12 and total bacteria. The primers and probes for these assays are detailed in Table 1. For the EvaGreen assays, the reaction volumes of $20 \mu \mathrm{L}$ contained $1 \times$ HOT FIREpol EvaGreen (Solis BioDyne, Tartu, Estonia), forward and reverse primers with a final concentration of $0.2 \mu M$ and $1 \mu \mathrm{L}$ of template DNA. A LightCycler-480 machine (Roche, Basel, Switzerland) was used for the $\mathrm{qPCR}$ reaction using the following settings: heating to $95^{\circ} \mathrm{C}$ for $15 \mathrm{~min}$, followed by 40 cycles of denaturing at $95^{\circ} \mathrm{C}$ for $15 \mathrm{~s}$, annealing and extension at $60^{\circ} \mathrm{C}$ for $1 \mathrm{~min}$. Melting point curves were obtained by heating the samples from 60 to $95^{\circ} \mathrm{C}$ at $0.1^{\circ} \mathrm{C}$ per second and measuring fluorescence at each $0.2^{\circ} \mathrm{C}$ temperature increment. For the TaqMan assay, the reaction volume of $20 \mu \mathrm{L}$ contained $4 \mu \mathrm{L}$ of $1 \times$ HOT FIREpol, $0.4 \mu \mathrm{L}$ of forward and reverse primers with a final concentration of $0.2 \mu \mathrm{M}$, the probe with a final concentration of 250 $\mathrm{n} M$ and $1 \mu \mathrm{L}$ of template DNA. The LightCycler- 480 settings for this assay were heating to $95^{\circ} \mathrm{C}$ for $12 \mathrm{~min}$, followed by 50 cycles of denaturing at $95^{\circ} \mathrm{C}$ for $30 \mathrm{~s}$, annealing and extension at $60^{\circ} \mathrm{C}$ for $1 \mathrm{~min}$. For both the EvaGreen and TaqMan assays, the negative control was $1 \mu \mathrm{L}$ of nuclease-free water and the positive control was DNA isolated from Biola or Bb12 cultures supplied by Tine AS (the producer of Biola) for the measurement of the administered bacteria and $1 \mu \mathrm{L}$ of Escherichia coli DNA for the analysis of total bacteria.

The general microbial composition of the samples was assessed using 16S rRNA gene sequencing of 300bp paired end reads on the Illumina MiSeq platform (Illumina Inc., San Diego, CA) with v3 sequencing chemistry at The Centre for Integrative Genetics (CIGENE, Ås, Norway). Following DNA extraction, the 
Table 1. Primers and probes

\begin{tabular}{llll}
\hline Bacteria $^{1}$ & Assay $^{2}$ & Forward/reverse primer and probe name & DNA sequence $\left(5^{\prime}-3^{\prime}\right)$ \\
\hline Total & TaqMan & Forward primer: Univ_F & Reverse primer: Univ_R \\
& & Probe: universal probe & TCCTACGGGAGGCAGCAGT \\
& & GGACTACCAGGGTATCTAATCCTGTT \\
LGG & EvaGreen & Forward: L.rhamn1 & FAM-GTATTACCGCGGCTGCTGGCAC-TAMRA \\
& Reverse: L.rhamn2 & CAATCTGAATGAACAGTGTC \\
La-5 & EvaGreen & Forward: La-5-5L & TATCTTGACCAACTTGACG \\
& Reverse: La-5-5R & TTACGCCAGTCCAAGGGTAG \\
Bb-12 & TaqMan & Forward primer & CAGAATGCCCGCAAGTTATC \\
& & Reverse primer & AGA ACC ACG GCG GCG TC \\
& & Probe & CGC GGT CTT CTC GAG CAC T \\
& & FAM-TGC GCT CGC CGA CG-MGB \\
\hline
\end{tabular}

${ }^{1} \mathrm{LGG}=$ Lactobacillus rhamnosus $\mathrm{GG}$; La-5 = Lactobacillus acidophilus La-5; Bb-12 = Bifidobacterium animalis ssp. lactis $\mathrm{Bb}-12$.

${ }^{2}$ TaqMan (Thermo Fisher Scientific, Waltham, MA); EvaGreen (Biotium Inc., Fremont, CA).

V3 to V4 region of the $16 \mathrm{~S}$ rRNA gene was amplified using PRK341F/PRK806R primers adapted for Illumina sequencing. The results were analyzed with the Quantitative Insights Into Microbial Ecology (QIIME) pipeline, utilizing AmpliconNoise to denoise the data and remove chimeras, uclust for operational taxonomic unit (OTU) clustering at $97 \%$ similarity and the Ribosomal Database Project classifier for assigning taxonomic identity to the identified OTU. A total of $1,712,182$ sequences were generated, with a median of 4,517.6 reads per sample.

\section{Statistical Analysis}

Statistical analyses and graphics were conducted using Stata IC 13.1 (StataCorp, College Station, TX) and MATLAB 2016b (The MathWorks Inc., Natick, MA). The effect of probiotic supplementation on the presence of the administered bacteria in breast milk at each time point was estimated using the $\mathrm{qPCR}$ data and is presented as a risk ratio (RR) and $95 \%$ confidence interval, accompanied with a Fisher exact test. Samples were considered positive if the EvaGreen assays had a threshold cycle $(\mathbf{C t})$ value below 35 and a melting point of $86^{\circ} \mathrm{C}$ for LGG or $80^{\circ} \mathrm{C}$ for La-5. For Bb- 12 and total bacteria, samples were considered positive if they had a Ct value below 35 on the TaqMan assay.

The 16S rRNA gene sequencing data was rarefied to 1,000 reads before descriptive and comparative analysis of the breastfeeding-associated microbiota. Samples with less than 1,000 reads were not included in these analyses. The effect of probiotic supplementation on the presence and relative abundance of individual genera at each time point was assessed using a $\chi^{2}$ test (or a Fisher exact test as indicated) and a Wilcoxon rank-sum test, respectively. An OTU was considered "present" if it accounted for at least $1 \%$ of reads (10 reads) in a given sample in the rarefied data set. To avoid excessive multiple comparisons of uncommon or very low abundance OTU, comparisons were only estimated for OTU and genera that were present in at least $10 \%$ of the samples (i.e., $\geq 10$ reads in $\geq 6$ samples from $10 \mathrm{~d}$ or $\geq 9$ samples from $3 \mathrm{mo}$ ). For each set of comparisons, a raw $P$-value of $\leq 0.05$ was considered of potential interest. False discovery rates (FDR) were obtained using the Benjamini-Hochberg method in the multiproc Stata command and an FDR $<0.1$ was considered acceptable. Associations between the presence or relative abundance of the common genera and the following factors were also considered: stage of lactation, the development of atopic dermatitis, presence of older siblings, mode of delivery, maternal atopy, and sex of the child. The association between the presence of common genera and the development of atopic dermatitis was also assessed using multivariable logistic regression models including treatment allocation, presence of older siblings, maternal atopy, and sex of the child as additional covariates.

The effect of probiotic supplementation and stage of lactation on the $\alpha$ diversity was assessed using the Shannon and Simpson diversity indices, phylogenetic diversity, and species richness (observed species). Beta diversity was investigated using principal coordinates analysis (PCoA) derived from unweighted and weighted UniFrac, distance matrices, and Bray Curtis dissimilarity matrix. Each PCoA plot was reviewed with respect to stage of lactation, treatment allocation, and the previously mentioned mother-infant characteristics.

\section{RESULTS}

\section{Participants}

A total of 472 breast milk samples from 252 women were available for analysis (Figure 1). Due to low quantities of bacterial DNA, fewer samples could be assessed using 16S rRNA gene sequencing (Figure 1). The baseline family characteristics and allergy-related disease outcomes among children in the current study are representative of the original ProPACT study population 
Table 2. Baseline characteristics of families and allergy-related disease in the children at $2 \mathrm{yr}$

\begin{tabular}{|c|c|c|c|c|c|}
\hline \multirow[b]{3}{*}{ Item } & \multicolumn{4}{|c|}{ Treatment allocation } & \multirow{3}{*}{$\begin{array}{l}\text { Risk ratio } \\
(95 \% \mathrm{CI})\end{array}$} \\
\hline & \multicolumn{2}{|c|}{ Probiotic } & \multicolumn{2}{|c|}{ Placebo } & \\
\hline & $\mathrm{n}$ & Value & $\mathrm{n}$ & Value & \\
\hline \multicolumn{6}{|l|}{ Characteristics } \\
\hline Age, mother, yr mean (SD) & 127 & $30.5(3.9)$ & 125 & $30.4(4.1)$ & \\
\hline Sex (male), child, no. (\%) & 127 & $66(52.0)$ & 125 & $51(40.8)$ & \\
\hline Siblings, no. (\%) & 127 & $60(47.2)$ & 125 & $53(42.4)$ & \\
\hline Atopy in family, no. (\%) & 127 & $90(70.9)$ & 125 & $92(73.6)$ & \\
\hline Maternal atopy, no. (\%) & 127 & $58(45.7)$ & 124 & $65(52.4)$ & \\
\hline Maternal smoking, ${ }^{1}$ no. $(\%)$ & 127 & $8(6.3)$ & 125 & $11(8.8)$ & \\
\hline Pet, ${ }^{2}$ no. $(\%)$ & 127 & $33(26.0)$ & 125 & $35(28.0)$ & \\
\hline Compliant, ${ }^{3}$ no. $(\%)$ & 123 & $111(90.2)$ & 123 & $110(89.4)$ & \\
\hline Mode of delivery, vaginal, no. (\%) & 123 & $108(87.8)$ & 124 & $108(87.1)$ & \\
\hline \multicolumn{6}{|l|}{ Allergy-related disease at $2 \mathrm{yr}$} \\
\hline Atopic dermatitis, ${ }^{4}$ no. $(\%)$ & 127 & $29(22.8)$ & 125 & $43(34.4)$ & $0.66(0.44-0.99)$ \\
\hline Current asthma, no. (\%) & 127 & $7(5.5)$ & 125 & $12(9.6)$ & $0.57(0.23-1.41)$ \\
\hline $\mathrm{ARC}^{5}$ no. $(\%)$ & 123 & $1(0.8)$ & 124 & $0(0.0)$ & NA \\
\hline Current sensitization, ${ }^{6}$ no. $(\%)$ & 120 & $18(15.0)$ & 119 & $14(11.7)$ & $1.28(0.67-2.44)$ \\
\hline Skin prick test positive, no. (\%) & 109 & $6(5.5)$ & 106 & $6(5.7)$ & $0.97(0.32-2.92)$ \\
\hline sIgE positive, no. (\%) & 80 & $17(21.3)$ & 89 & $12(13.5)$ & $1.58(0.80-3.09)$ \\
\hline \multirow{2}{*}{\multicolumn{6}{|c|}{${ }^{1}$ Maternal smoking reported during pregnancy, or 6 wk or 12 mo postpartum. }} \\
\hline & & \multicolumn{4}{|c|}{${ }^{2}$ Reported a household pet during pregnancy or the child's first year of life. } \\
\hline \multicolumn{6}{|c|}{$\begin{array}{l}{ }^{3} \text { Compliance with the study protocol was defined as consumption of the study milk on at least } 50 \% \text { of days } \\
\text { from } 36 \text { wk gestation to } 12 \text { postpartum, no consumption of other products with probiotics and at least partial } \\
\text { breastfeeding until } 3 \text { mo postpartum. }\end{array}$} \\
\hline \multicolumn{6}{|c|}{${ }^{4}$ Cumulative incidence of atopic dermatitis. } \\
\hline \multicolumn{6}{|c|}{${ }^{5} \mathrm{ARC}=$ allergic rhinoconjunctivitis (cumulative incidence). } \\
\hline \multicolumn{6}{|c|}{$\begin{array}{l}\left.{ }^{6} \text { Allergic sensitization defined as positive skin prick test (wheal } \geq 3 \mathrm{~mm}\right) \text { and or positive } \operatorname{sIgE}\left(\geq 0.35 \mathrm{kUL}^{-1}\right) \\
\text { Not all children had both measured. }\end{array}$} \\
\hline
\end{tabular}

(Table 2 and Supplemental Table S1; https://doi.org/ 10.3168/jds.2017-13411). The baseline characteristics of the families with adequate reads on $16 \mathrm{~S}$ rRNA gene sequencing analysis were also comparable; however, the preventative effect of probiotic supplementation on atopic dermatitis was not observed in this subgroup (Supplemental Table S1; https://doi.org/10.3168/jds .2017-13411).

\section{Transfer of Administered Probiotic Bacteria Strains Via Breast Milk}

Based on results from the qPCR assays, the majority of the breast milk samples had measurable quantities of bacterial DNA with 392 of 472 samples positive for total bacteria. Samples without measurable bacteria included 39 samples collected at 10 d postpartum (20 and 19 from the probiotic and placebo groups, respectively) and 41 samples from 3 mo postpartum (26 and 15 from the probiotic and placebo group, respectively). However, only 8 women from the probiotic group and 1 from the placebo group had detectable levels of the any of the administered bacteria. At $10 \mathrm{~d}$ postpartum, LGG was present in 2 of 114 samples collected from women in the probiotic group and 1 of 121 samples from the placebo group (RR 4.2, 95\% CI 0.5-36.8, Fisher's exact $P=0.21)$. The La-5 was detected in 2 samples from the probiotic group only at $10 \mathrm{~d}$ postpartum (RR not applicable, $P=0.49)$. Neither LGG nor La-5 were found in breast milk samples collected 3 mo postpartum $(\mathrm{n}=$ 120 and 117 for the probiotic and placebo groups, respectively). The Bb-12 was present in breast milk samples from women in the probiotic group only, including 5 samples at $10 \mathrm{~d}$ postpartum (RR not applicable, $P=$ 0.03 ) and 2 samples at 3 mo postpartum (RR not applicable, $P=0.50)$. Two women with Bb-12 in breast milk at $10 \mathrm{~d}$ also had one of the other administered bacteria in their milk sample, one with LGG and one with La-5. Additionally, the woman with both LGG and Bb12 at $10 \mathrm{~d}$ was also found to have Bb12 in her breast milk at 3 mo. Given that so few samples were positive for the administered probiotic bacteria, comparison of the relative abundance of these bacteria between the probiotic and placebo groups was not feasible. Similarly, it was not feasible to conduct any formal statistical assessment of the association between the presence of the administered bacteria in breast milk samples and previously analyzed mother and infant stool samples (Dotterud et al., 2015) or the development of atopic dermatitis in infants. A summary of these results for 
mother-infant pairs with positive breast milk samples are presented in Supplemental Table S2 (https://doi .org/10.3168/jds.2017-13411).

\section{Breastfeeding-Associated Microbiota at Different Stages of Lactation}

Due to low quantity and quality of bacterial DNA in the breast milk samples, not all samples were sequenced and fewer samples had sufficient reads to be included in further analysis. Samples that were removed before sequencing had a median $\mathrm{Ct}$ value for total bacteria of 33.4 [interquartile range (IQR) 31.9-36.1, $\mathrm{n}=128$ ] and samples that were sequenced, but had insufficient reads, had a median Ct value of 31.7 (IQR 29.7-33.8, $\mathrm{n}=199)$. Ultimately, sufficient reads were obtained for 142 samples from 125 women (median $\mathrm{Ct}$ value 30.2, IQR 27.9-32.0). These reads were attributed to a total of 307 OTU belonging to 147 genera. Considering the composition of the milk microbiota at the genus level, 45 and 69 genera were present ( $\geq 10$ reads) in samples collected at $10 \mathrm{~d}$ and $3 \mathrm{mo}$, respectively. However, only 11 genera were present in at least $10 \%$ of samples at either time point and no genus was present in all samples (Table 3 and Supplemental Table S3 for OTU level summary; https://doi.org/10.3168/jds .2017-13411). Even at the phyla level, no phylum was present in all breast milk samples, although Firmicutes bacteria were present in all but one 3-mo sample (data not shown). The general breastfeeding-associated microbiota at $10 \mathrm{~d}$ and 3 mo postpartum was dominated by Streptococcus and Staphylococcus genera in most women (Supplemental Figure S1; https://doi.org/10 $.3168 /$ jds.2017-13411). At both time points, a subgroup of women had a milk microbiota dominated by other genera, such as Acinetobacter and genera from the Bacillaceae family (Supplemental Figure S1). Additionally, some individual women had moderate to high relative abundances of uncommon genera, such as Klebsiella, Lactobacillus zeae, Alkanindeges, Stenotrophononas, and genera from the Caulobacteraceae family (data not shown, these genera fall into the category of "other" in Supplemental Figure S1).

When assessing the influence of the stage of lactation on specific genera, we found a higher presence and relative abundance of Staphylococcus in 10-d compared with 3 -mo samples (Table 3 and Figure 2a). The samples from $10 \mathrm{~d}$ postpartum also had a lower prevalence and relative abundance of Rothia and Veillonella genera and a higher relative abundance of unassigned bacteria in 10-d samples. The Granulicatella and Methylbacterium had higher prevalence in 3-mo samples and Granulicatella had a higher relative abundance. The number of observed species, phylogenetic diversity, and Shannon's

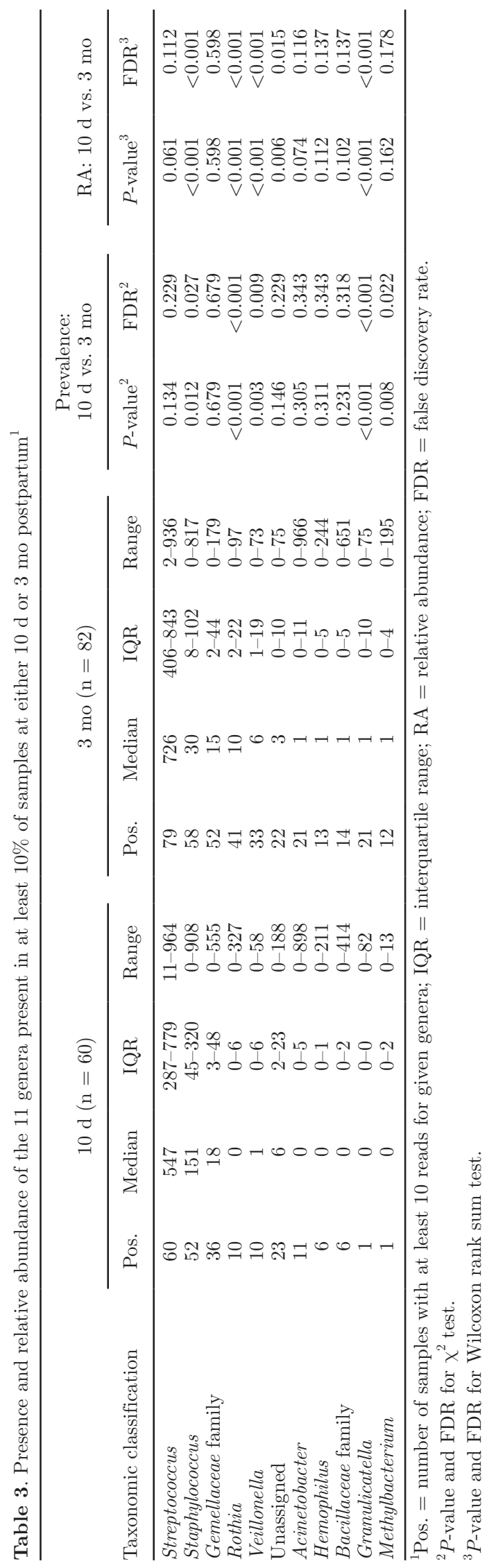




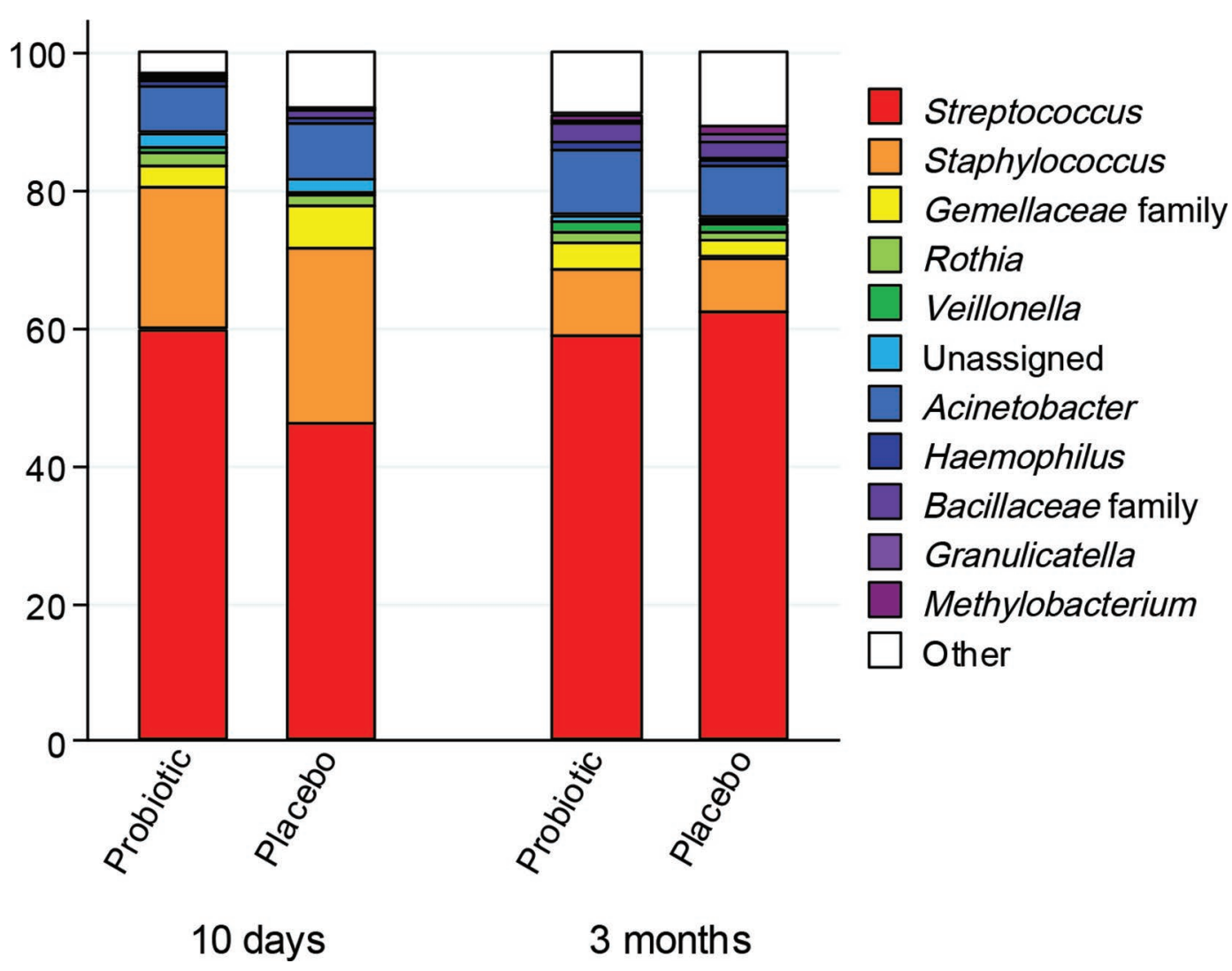

Figure 2. Overview of proportion of reads attributable to the 11 genera present in at least $10 \%$ of samples at $10 \mathrm{~d}$ or 3 mo in the probiotic and placebo groups.

diversity index of the breastfeeding-associated microbiota increased from $10 \mathrm{~d}$ to 3 mo postpartum (Shannon's index median (IQR): $1.70(1.08-2.42)$ vs $2.15(1.63-$ 2.47), $P=0.016$, Supplemental Figure S2; https://doi .org/10.3168/jds.2017-13411). The PCoA plots based on weighted and unweighted UniFrac distance matrices and Bray Curtis dissimilarity matrix did not indicate a clear separation in the breastfeeding-associated microbiota by stage of lactation (Supplemental Figure S3; https://doi.org/10.3168/jds.2017-13411), although 10 -d samples were statistically more likely to be associated divergent microbiotas in the unweighted UniFrac and Bray Curtis analyses (data not shown).

An adequate number of reads was achieved in both 10-d and 3-mo samples from 17 women (Supplemental Figure S4; https://doi.org/10.3168/jds.2017-13411). Individually, these women had between 5 and 17 OTU (median 10, IQR 7-11) represented at either time point, with between 0 and 5 OTU present in both samples (median 3, IQR 2-4). These persistent OTU accounted for a large proportion of total reads in most women: median of $80.0 \%$ (IQR 36.7-93.2\%, range 0.0-99.4\%) of the total reads at $10 \mathrm{~d}$ and $72.5 \%$ (31.8-85.0, range $0.0-92.8$ ) of the reads at 3 mo. However, 6 of 17 women had substantial changes in the OTU-level composition of their breast milk microbiota, with the persistent OTU accounting for less than $40 \%$ of reads at either or both time points. One of these women had no persistent OTU, suggesting a total replacement of the breastfeeding-associated microbial community between $10 \mathrm{~d}$ and $3 \mathrm{mo}$ (M10 in Figure S4; https://doi.org/10 $.3168 /$ jds.2017-13411).

At each time point, probiotics had no statistically significant effect on the presence or relative abundance of any of the individual genera that were present in at least $10 \%$ of the samples (Supplemental Table S4, https://doi.org/10.3168/jds.2017-13411, and Figure 2). Furthermore, probiotic supplementation was not found to have a statistically significant effect on the $\alpha$ or $\beta$ diversity of the breast milk microbiota at either time point (Supplemental Figures S2 and S3; https://doi .org/10.3168/jds.2017-13411). In the subgroup of 17 women with $16 \mathrm{~S}$ rRNA gene sequencing data, probiotic supplementation may have a positive influence on the stability of the breastfeeding-associated microbiota (RR of stable breastfeeding-associated microbiota after probiotic supplementation: 2.37, 95\% CI 0.94-5.97, Fisher's exact $P=0.050$ ). 


\section{Breastfeeding-Associated Microbiota and Atopic Dermatitis in Offspring}

Finally, we considered the association between the general breastfeeding-associated microbiota and the development of atopic dermatitis. The presence of the Staphylococcus and Veillonella genera at 3 mo postpartum tended to be associated with a borderline greater risk of developing atopic dermatitis, although the FDR for these comparisons was unacceptably high (RR 1.93, 95\% CI 0.92-4.06, $P=0.052$ for Staphylococcus; RR 1.67, 95\% CI 1.00-2.78, $P=0.048$ for Veillonella; and $\mathrm{FDR}=0.285$ for both). Similarly, a higher relative abundance of Staphylococcus at 3 mo appeared to increase the risk of atopic dermatitis before considering the FDR [median number of reads per 1,000 (IQR): 40 (18-128) vs. 14 (5-63) for children with and without atopic dermatitis, respectively, $P=0.045$, FDR: 0.50]. No other associations were found between the development of atopic dermatitis and the presence or relative abundance of the common genera in 10-d or 3-mo breast milk samples (data not shown). The relationship between the breastfeeding-associated microbiota and the development of atopic dermatitis is potentially confounded by other maternal-infant characteristics, such as the presence of older siblings, mode of delivery, maternal atopy, and the sex of the child. The Gemellaceae family was more common in 10-d samples from mothers with a personal history of allergy (23/31 vs. $13 / 29, P=0.020$, FDR $=0.081)$, whereas the Rothia genera was more commonly observed in 10-d samples from mothers with no allergic history ( $9 / 29$ vs. $1 / 31, P$ $=0.004, \mathrm{FDR}=0.031)$. Samples collected at $3 \mathrm{mo}$ from mothers of female infants also had a higher presence and relative abundance of the Rothia genera compared with mothers of male infants (present in $30 / 48$ vs. 11/34 for female and male infants, respectively, $P=0.007$, FDR $=0.079$, and median (IQR): $14(5-27)$ vs. $4(0-12)$, respectively, $P=0.005$, FDR $=0.055)$. Also at $3 \mathrm{mo}$ postpartum, Streptococcus was more commonly present in breast milk samples after vaginal delivery, although the FDR was unacceptably high $(65 / 66$ vs. $23 / 14$ in vaginal vs. cesarean delivery, $P=0.022, \mathrm{FDR}=0.246)$. No other statistically significant associations between mother-infant characteristics and the presence or relative abundance of individual bacterial genera were observed at either time point (data not shown). The presence and relative abundance of the Staphylococcus and Veillonella genera, which were highlighted for their potential association with the development of atopic dermatitis, do not appear to be related to any of these maternal-infant characteristics. Further, the strength of association between the presence of individual bacterial genera and the development of atopic dermatitis was not significantly altered by the addition of these maternal-infant characteristics as covariates in a multivariable logistic regression models (data not shown). Neither the development of atopic dermatitis nor these mother-infant characteristics were associated with a clear separation of samples when reviewing the PCoA plots, suggesting that the $\beta$-diversity of these samples was not defined by these characteristics (Supplemental Figure S3; https://doi.org/10.3168/jds.2017-13411).

\section{DISCUSSION}

Our findings indicate that pre- and postnatal maternal probiotic supplementation with LGG, La-5, and $\mathrm{Bb}-12$ leads to the presence of these bacteria in breast milk samples for only a small subgroup of women. Thus, breast milk was not a meaningful source of LGG, La-5, or Bb-12 for the infants in the ProPACT trial, and the observed preventative effect of the ProPACT regimen on atopic dermatitis cannot be attributed to the transfer of these bacteria through breastfeeding. Using $16 \mathrm{~S}$ rRNA gene sequencing, we also demonstrate that the general breastfeeding-associated microbiota of human milk is largely dominated by Streptococcus and Staphylococcus genera at both $10 \mathrm{~d}$ and 3 mo postpartum and that maternal probiotic supplementation does not have a substantial effect on this microbiota or its diversity.

One of the major strengths of this study is the use of samples collected in a randomized, placebo controlled trial with clinical follow-up. This allows us to conduct an unbiased assessment of the effect of probiotic supplementation on the presence of administered bacteria and the breastfeeding-associated microbiota. Furthermore, the qPCR analysis has a high sensitivity and specificity for the administered bacteria. Therefore, with only 9 of 252 women found to have any administered bacteria in their breast milk at either time point, breast milk is unlikely to be a substantial source of LGG, La-5, or Bb-12 for infants after maternal supplementation. Although we found a statistically significant increase in the presence of $\mathrm{Bb}-12$ at $10 \mathrm{~d}$ postpartum in the probiotic group, over $95 \%$ of the samples had no detectable Bb-12 and we do not consider this statistical significance to imply a clinically meaningful transfer of Bb-12 during breastfeeding. In a previous study, we found that as many as 50 out of $129(39 \%)$ infants in the probiotic group had LGG present in their stool samples at $10 \mathrm{~d}$ of age and 56 of $122(46 \%)$ at 3 mo of age. This represented a statistically significant increase in the presence of LGG, which was found in only 7 of 133 $(5 \%)$ and 23 of $121(19 \%)$ stool samples from infants in the placebo group at $10 \mathrm{~d}$ and 3 mo, respectively. In contrast, the current study identified LGG in only 3 breast milk samples collected at $10 \mathrm{~d}$ postpartum 
and none at $3 \mathrm{mo}$, suggesting that breastfeeding cannot have been the source of LGG for the majority of infants who had this bacteria present in their stool samples.

In previous randomized studies of maternal supplementation, Lactobacillus species have been recovered from breast milk in 12 to $53 \%$ of women after supplementation (Abrahamsson et al., 2009; Arroyo et al., 2010; Nasiraii et al., 2011; Hurtado et al., 2017). In a small trial, 4 of 10 women had viable cultures of $L$. rhamnosus LC705 isolated in breast milk samples after supplementation with that probiotic (Nasiraii et al., 2011). Also using culture-based techniques, Abrahamsson et al. (2009) demonstrated that $12 \%$ of women in their probiotic group had viable $L$. reuteri in colostrum samples, compared with $2 \%$ in the placebo group. Similarly, in a 3-arm trial, Arroyo et al. (2010) investigated L. fermentum CECT5716, L. salivarius CECT5713 or antibiotics in the treatment of mastitis and found that just over $50 \%$ of women in both probiotic arms, and none in the antibiotic arm, had lactobacilli cultured in breast milk samples after $21 \mathrm{~d}$ of supplementation. Results from subsequent trials suggest that maternal supplementation with $L$. fermentum CECT5716 may increase levels of that bacteria (Hurtado et al., 2017), but not the overall abundance of lactobacilli (Maldonado-Lobon et al., 2015; Hurtado et al., 2017). It is worth noting that both L. fermentum CECT5716, and L. salivarius CECT5713 were initially isolated from human milk samples, suggesting that these bacteria have a natural affinity for the breast milk microbiota. The low proportion of milk samples with either LGG, La-5, or Bb-12 in the current study may reflect strain-specific differences in their ability to be transferred to the milk microbiota. We also found that maternal probiotic supplementation did not significantly affect the general breastfeeding-associated microbiota, which was investigated using $16 \mathrm{~S}$ rRNA gene sequencing. Furthermore, the development of atopic dermatitis was not convincingly associated with individual genera or the overall composition of this microbiota.

Over the past 5 yr, several studies have employed sequencing methods to characterize the breast milk or breastfeeding-associated microbiota and its association with mother-infant characteristics such as stage of lactation (Hunt et al., 2011; Cabrera-Rubio et al., 2012; Jost et al., 2013; Sakwinska et al., 2016; Urbaniak et al., 2016; Murphy et al., 2017), prematurity (Urbaniak et al., 2016), mode of delivery and elective versus nonelective caesarean section deliveries (Cabrera-Rubio et al., 2012, 2016; Kumar et al., 2016; Sakwinska et al., 2016; Urbaniak et al., 2016), infant sex (Urbaniak et al., 2016), maternal weight (Cabrera-Rubio et al., 2012; Collado et al., 2012), geography (Kumar et al., 2016; Li et al., 2017), and mastitis (Jiménez et al., 2015).
Each of these mother-infant characteristics has been described to influence the microbiota composition, particularly in the smaller studies $(\mathrm{n}=7-20)$. Three of the larger studies $(\mathrm{n}=133, \mathrm{n}=90$, and $\mathrm{n}=39)$ report no clear distinction in the breast milk microbiota based on stage of lactation, prematurity, or mode of delivery (Sakwinska et al., 2016; Urbaniak et al., 2016; Li et al., 2017). Our results suggest that stage of lactation, sex, maternal atopy, and mode of delivery may influence the presence or relative abundance of specific genera. However, neither these characteristics, nor the presence of siblings, defined the overall composition of the breastfeeding-associated microbiota. Due to lack of information, we were unable to assess the effect of maternal antibiotic use and signs or symptoms of mastitis. Information regarding antibiotic use and the presence of mastitis would have been particularly useful with respect to the observation that probiotic supplementation may promote stability of the breastfeeding-associated microbiota.

Another recurrent theme among some of the previous smaller studies is the presentation of a "core" breast milk microbiota of between 6 and 12 OTU or genera, which are found in 90 to $100 \%$ of samples (Hunt et al., 2011; Jiménez et al., 2015; Murphy et al., 2017). In the present study we did not observe a "core" breastfeeding-associated microbiota, despite participants coming from a small geographic area. This is likely due to the high degree of individual variability and relatively large number of women included in our study. Indeed, the lack of a "core" microbiota is consistent with the conclusion of a recent systematic review, which identified Staphylococcus and Streptococcus as the only genera to be repeatedly reported as dominant breast milk-related genera (Fitzstevens et al., 2017). Methodological differences in sample collection and laboratory analysis are likely to influence the observed microbiota in our study and other published studies.

Our $16 \mathrm{~S}$ rRNA gene sequencing data probably represent a combination of the breast milk and breast surface microbiotas because women were not provided with instructions for sterile collection. This has presumably resulted in contamination from the skin microbiota and possibly the infant oral microbiota, and may account for the higher relative abundance of Staphylococcus and Streptococcus genera in our results compared with previous sequencing studies that have used sterile collection protocols. Indeed, Sakwinska et al. (2016) demonstrate that the method of collection significantly influences the microbiota composition, although it was the higher presence and relative abundance of Acinetobacter species that appeared to characterize the nonsterile samples in their study. Due to the nonsterile sample collection method, we consider our results more rep- 
resentative of the microbiota transferred while breastfeeding, rather than what infants receive from breast milk alone. We note here that we presume the relative abundance, but not the presence, of the administered bacteria would have been affected by the nonsterile collection. We therefore believe that the collection method is unlikely to have masked a significant transfer of the administered bacteria via breast milk and we consider the qPCR results to be a clear indication that breastfeeding was not a substantial route of probiotic transfer in the ProPACT study. These findings would also suggest that, even if probiotic supplementation altered the breast milk microbiota, the overall composition of the microbiota transferred during breastfeeding was not statistically significantly altered. The results of the $16 \mathrm{~S}$ rRNA gene sequencing are also limited because only $30 \%$ of samples had enough reads to be included in further analysis and only 17 individuals had adequate reads at both time points. The baseline characteristics of the women with successful sequencing was representative of the original ProPACT population, and with 142 successfully sequenced samples, this currently represents the largest sequencing study of the breastfeeding-associated microbiota. Nonetheless, due to the high degree of individual variation, large studies are required to further investigate this microbiota and its association with mother-infant characteristics and infant health outcomes.

This study was motivated by the findings of the ProPACT trial, namely, that maternal probiotic supplementation reduced the cumulative incidence of atopic dermatitis in offspring at 2 yr (Dotterud et al., 2010) and led to a higher prevalence and relative abundance of LGG in infant stool samples up to 3 mo of age (Dotterud et al., 2015). In adults, continuous ingestion of LGG is required to maintain measurable quantities in stool samples (Alander et al., 1999). Given that ongoing transfer does not appear to come from breastfeeding, the observation of LGG in infant stool samples at $3 \mathrm{mo}$ of age suggests that either early transfer is sufficient to establish a stable colonization with LGG in the relatively microbe-free newborn gut, or that children are receiving continued transfer of LGG via some other route from their mother. On a technical note, due to the low quantities of bacterial DNA in breast milk samples, future studies of the breast milk or breastfeeding-associated microbiota should consider starting with a greater volume of milk and establishing methods to maximize DNA extraction from milk.

In conclusion, we find that maternal supplementation with LGG, La5, and Bb-12 does not result in substantial transfer of these bacteria via breastfeeding. The breastfeeding-associated microbiota is largely domi- nated by Streptococcus and Staphylococcus genera at both $10 \mathrm{~d}$ and 3 mo postpartum.

\section{ACKNOWLEDGMENTS}

We thank all the families for their participation in the ProPACT study, the midwives and general practitioners of the Trondheim region who recruited them, Rakel Berg (pediatrician, retired, St. Olavs University Hospital, Trondheim, Norway), who assessed the children at the 2-year follow-up, and the project assistants who coordinated the follow-up and collection of samples [Guri Helmersen (NTNU) and Else Bartnes (St. Olavs University Hospital)]. We also acknowledge technical assistance of Ida Kristin Myhrvold and Inga Leena Angell (University of Life Sciences) in the isolation of DNA and the qPCR analysis, and Agnieszka Segin (University of Life Sciences) for the 16S rRNA gene sequencing laboratory analyses.

\section{REFERENCES}

Abrahamsson, T. R., G. Sinkiewicz, T. Jakobsson, M. Fredrikson, and B. Bjorksten. 2009. Probiotic lactobacilli in breast milk and infant stool in relation to oral intake during the first year of life. J. Pediatr. Gastroenterol. Nutr. 49:349-354. https://doi.org/10.1097/ MPG.0b013e31818f091b.

Alander, M., R. Satokari, R. Korpela, M. Saxelin, T. VilpponenSalmela, T. Mattila-Sandholm, and A. von Wright. 1999. Persistence of colonization of human colonic mucosa by a probiotic strain, Lactobacillus rhamnosus GG, after oral consumption. Appl. Environ. Microbiol. 65:351-354.

Arroyo, R., V. Martín, A. Maldonado, E. Jiménez, L. Fernández, and J. M. Rodríguez. 2010. Treatment of infectious mastitis during lactation: Antibiotics versus oral administration of lactobacilli isolated from breast milk. Clin. Infect. Dis. 50:1551-1558. https://doi . org $/ 10.1086 / 652763$.

Bäckhed, F., J. Roswall, Y. Peng, Q. Feng, H. Jia, P. KovatchevaDatchary, Y. Li, Y. Xia, H. Xie, H. Zhong, M. T. Khan, J. Zhang, J. Li, L. Xiao, J. Al-Aama, D. Zhang, Y. S. Lee, D. Kotowska, C. Colding, V. Tremaroli, Y. Yin, S. Bergman, X. Xu, L. Madsen, K. Kristiansen, J. Dahlgren, and J. Wang. 2015. Dynamics and stabilization of the human gut microbiome during the first year of life. Cell Host Microbe 17:690-703. https://doi.org/10.1016/j .chom.2015.04.004.

Cabrera-Rubio, R., M. C. Collado, K. Laitinen, S. Salminen, E. Isolauri, and A. Mira. 2012. The human milk microbiome changes over lactation and is shaped by maternal weight and mode of delivery. Am. J. Clin. Nutr. 96:544-551. https://doi.org/10.3945/ajcn.112 .037382 .

Cabrera-Rubio, R., L. Mira-Pascual, A. Mira, and M. C. Collado. 2016. Impact of mode of delivery on the milk microbiota composition of healthy women. J. Dev. Orig. Health Dis. 7:54-60. https:// doi.org/10.1017/S2040174415001397.

Cacho, N. T., and R. M. Lawrence. 2017. Innate immunity and breast milk. Front. Immunol. 8:584. https://doi.org/10.3389/fimmu.2017 .00584 .

Collado, M. C., K. Laitinen, S. Salminen, and E. Isolauri. 2012. Maternal weight and excessive weight gain during pregnancy modify the immunomodulatory potential of breast milk. Pediatr. Res. 72:77-85. https://doi.org/10.1038/pr.2012.42.

Dotterud, C. K., E. Avershina, M. Sekelja, M. R. Simpson, R. Knut, O. Storrø, R. Johnsen, and T. Øien. 2015. Does maternal perinatal 
probiotic supplementation alter the intestinal microbiota of mother and child? A randomised controlled trial. J. Ped. Gastroenterol. Nutr. https://doi.org/10.1097/MPG.0000000000000781.

Dotterud, C. K., O. Storro, R. Johnsen, and T. Oien. 2010. Probiotics in pregnant women to prevent allergic disease: A randomized, double-blind trial. Br. J. Dermatol. 163:616-623. https://doi.org/ 10.1111/j.1365-2133.2010.09889.x.

Fitzstevens, J. L., K. C. Smith, J. I. Hagadorn, M. J. Caimano, A. P. Matson, and E. A. Brownell. 2017. Systematic review of the human milk microbiota. Nutr. Clin. Pract. 32:354-364. https://doi .org/10.1177/0884533616670150.

Hunt, K. M., J. A. Foster, L. J. Forney, U. M. Schutte, D. L. Beck, Z. Abdo, L. K. Fox, J. E. Williams, M. K. McGuire, and M. A. McGuire. 2011. Characterization of the diversity and temporal stability of bacterial communities in human milk. PLoS One 6:e21313. https://doi.org/10.1371/journal.pone.0021313.

Hurtado, J. A., J. A. Maldonado-Lobón, M. P. Díaz-Ropero, K. FloresRojas, J. Uberos, J. L. Leante, L. Affumicato, M. L. Couce, J. M. Garrido, M. Olivares, and J. Fonollá. 2017. Oral administration to nursing women of Lactobacillus fermentum CECT5716 prevents lactational mastitis development: A randomized controlled trial. Breastfeed. Med. 12:202-209. https://doi.org/10.1089/bfm.2016 .0173 .

Jiménez, E., J. de Andrés, M. Manrique, P. Pareja-Tobes, R. Tobes, J. F. Martínez-Blanch, F. M. Codoñer, D. Ramón, L. Fernández, and J. M. Rodríguez. 2015. Metagenomic analysis of milk of healthy and mastitis-suffering women. J. Hum. Lact. 31:406-415. https:// doi.org/10.1177/0890334415585078.

Jost, T., C. Lacroix, C. Braegger, and C. Chassard. 2013. Assessment of bacterial diversity in breast milk using culture-dependent and culture-independent approaches. Br. J. Nutr. 110:1253-1262. https://doi.org/10.1017/S0007114513000597.

Kumar, H., E. du Toit, A. Kulkarni, J. Aakko, K. M. Linderborg, Y. Zhang, M. P. Nicol, E. Isolauri, B. Yang, M. C. Collado, and S. Salminen. 2016. Distinct patterns in human milk microbiota and fatty acid profiles across specific geographic locations. Front. Microbiol. 7:1619. https://doi.org/10.3389/fmicb.2016.01619.

Li, S. W., K. Watanabe, C. C. Hsu, S. H. Chao, Z. H. Yang, Y. J. Lin, C. C. Chen, Y. M. Cao, H. C. Huang, C. H. Chang, and Y. C. Tsai. 2017. Bacterial composition and diversity in breast milk samples from mothers living in Taiwan and mainland China. Front. Microbiol. 8:965. https://doi.org/10.3389/fmicb.2017.00965.

Maldonado-Lobon, J. A., M. A. Diaz-Lopez, R. Carputo, P. Duarte, M. P. Diaz-Ropero, A. D. Valero, A. Sanudo, L. Sempere, M. D. Ruiz-Lopez, O. Banuelos, J. Fonolla, and M. Olivares Martin. 2015. Lactobacillus fermentum CECT 5716 reduces Staphylococcus load in the breastmilk of lactating mothers suffering breast pain: A randomized controlled trial. Breastfeed. Med. 10:425-432. https:// doi.org/10.1089/bfm.2015.0070.

Murphy, K., D. Curley, T. F. O'Callaghan, C. A. O'Shea, E. M. Dempsey, P. W. O'Toole, R. P. Ross, C. A. Ryan, and C. Stanton.
2017. The composition of human milk and infant faecal microbiota over the first three months of life: A pilot study. Sci. Rep. 7:40597. https://doi.org/10.1038/srep40597.

Nasiraii, L. R., F. Tabatabaie, B. Aleaeddini, R. Noorbakhsh, R. M. Heravi, and S. Afsharian. 2011. Investigation of lactobacilli from mother's breast milk who were placed on probiotic diet. Afr. J. Microbiol. Res. 5:1581-1585. https://doi.org/10.5897/AJMR10.872.

Rodríguez, J. M. 2014. The origin of human milk bacteria: Is there a bacterial entero-mammary pathway during late pregnancy and lactation? Adv. Nutr. 5:779-784. https://doi.org/10.3945/an.114 .007229 .

Rodríguez, J. M., K. Murphy, C. Stanton, R. P. Ross, O. I. Kober, N. Juge, E. Avershina, K. Rudi, A. Narbad, M. C. Jenmalm, J R. Marchesi, and M. C. Collado. 2015. The composition of the gut microbiota throughout life, with an emphasis on early life. Microb. Ecol. Health Dis. 26:26050. https://doi.org/10.3402/mehd .v26.26050.

Sakwinska, O., D. Moine, M. Delley, S. Combremont, E. Rezzonico, P. Descombes, G. Vinyes-Pares, Y. Zhang, P. Wang, and S. K. Thakkar. 2016. Microbiota in breast milk of Chinese lactating mothers. PLoS One 11:e0160856. https://doi.org/10.1371/journal.pone .0160856 .

Simpson, M. R., C. K. Dotterud, O. Storrø, R. Johnsen, and T. Øien. 2015. Perinatal probiotic supplementaion in the prevention of allergy related disease: 6 year follow up of a randomised controlled trial. BMC Dermatol. 15:13. https://doi.org/10.1186/s12895-015 -0030-1.

Treven, P., V. Mrak, B. Bogovič Matijašić, S. Horvat, and I. Rogelj. 2015. Administration of probiotics Lactobacillus rhamnosus GG and Lactobacillus gasseri K7 during pregnancy and lactation changes mouse mesenteric lymph nodes and mammary gland microbiota. J. Dairy Sci. 98:2114-2128. https://doi.org/10.3168/jds .2014-8519.

Urbaniak, C., M. Angelini, G. B. Gloor, and G. Reid. 2016. Human milk microbiota profiles in relation to birthing method, gestation and infant gender. Microbiome 4:1. https://doi.org/10.1186/ s40168-015-0145-y.

Williams, H. C., P. G. Burney, R. J. Hay, C. B. Archer, M. J. Shipley, J. J. Hunter, E. A. Bingham, A. Y. Finlay, A. C. Pembroke, R. A. Graham-Brown, D. A. Atherton, M. S. Lewis-Jones, C. A. Holden, J. I. Harper, R. H. Champion, T. F. Poyner, J. Launer, and T. J. David. 1994. The U.K. Working Party's Diagnostic Criteria for Atopic Dermatitis. I. Derivation of a minimum set of discriminators for atopic dermatitis. Br. J. Dermatol. 131:383-396. https:// doi.org/10.1111/j.1365-2133.1994.tb08530.x.

Wopereis, H., R. Oozeer, K. Knipping, C. Belzer, and J. Knol. 2014. The first thousand days - intestinal microbiology of early life: Establishing a symbiosis. Pediatr. Allergy Immunol. 25:428-438. https://doi.org/10.1111/pai.12232. 